\title{
A Polarized Photobleaching Study of Chromatin Reorientation in Intact Nuclei
}

\author{
Paul R. Selvin ${ }^{1,2}$, Bethe A. Scalettar ${ }^{1}$, John P. Langmore ${ }^{3}$, Daniel Axelrod ${ }^{4}$, \\ Melvin P. Klein ${ }^{1}$ and John E. Hearst ${ }^{1,5}$ \\ ${ }^{1}$ Chemical Biodynamics Division \\ Lawrence Berkeley Laboratory \\ Berkeley, CA 94720, U.S.A. \\ ${ }^{2}$ Department of Physics and ${ }^{5}$ Department of Chemistry \\ University of California \\ Berkeley, CA 94720, U.S.A. \\ ${ }^{3}$ Department of Biology and ${ }^{4}$ Department of Physics \\ University of Michigan \\ Ann Arbor, MI 48109, U.S.A.
}

(Received 7 December 1989; accepted 1 May 1990)

\begin{abstract}
Polarized fluorescence recovery after photobleaching (pFRAP) was used to monitor the effects that condensation, i.c. compaction and aggrcgation, have on the ( $\mu$ s and ms) internal dynamics of chromatin in intact nuclei. When divalent cations were present with physiological $(\approx 90 \mathrm{~mm}$ ) monovalent salt the chromatin was found to exist in a compact and aggregated state which was characterized by rotational immobilization over timescales that range from 10 microseconds to 40 milliseconds. This immobilization is attributed to suppression of internal dynamics by intermolecular interactions. When the divalent cations were removed, the compact fibers no longer aggregated and were free to reorient with a characteristic decay time of about 1.2 milliseconds. It is shown that this millisecond relaxation could represent rigid rotation of topologically independent structural domains. Dilution of the monovalent salt induced a gradual change in the structural state of the chromatin that was manifest as a dramatic increase in internal flexibility. At the lowest salt concentration studied (11 mM-monovalent salt) the chromatin reorients in fewer than ten microseconds. These changes in flexibility are continuous with salt concentration, indicating that there are no well-defined endpoints to structural transitions and that the microsecondmillisecond internal dynamics of chromatin are a sensitive measure of structure. Measurements made on nuclei from cells that are either transcriptionally quiescent or active indicate that the dynamics mirrors biological activity.
\end{abstract}

\section{Introduction}

It is apparent that cell plasmas and membranes contain very high concentrations of biological macromolecules (for reviews, see Fulton, 1982; Kellenberger, 1987). Nevertheless, the (reconstituted) solutions that are used to study biological phenomena typically contain relatively dilute concentrations of cellular components. Therefore, it is important to determine what effects the very highly congested conditions that exist in vivo have on the behavior of biological macromolecules. Here we focus on understanding the relationship between the condensation state of chromatin and chromatin dynamies.
Chromatin provides a particularly striking example of the way in which biological function can be modulated by condensation state. For example, it is believed that metabolically inert and mitotic chromatin is stored in a very closely packed form. In contrast, actively transcribing and replicating chromatin is decondensed, facilitating interaction of macromolecular reactants and products (Kellenberger, 1987).

The condensation state of chromatin is generally believed to be influenced by two factors: the extent to which the individual molecules are compacted and the degree to which they are aggregated. The least compact form of chromatin is a "beads-on-astring" structure, which consists of a repeating 
array of particles known as nucleosomes (for a review, see Van Holde, 1989). Each nucleosome is composed of a core, which contains 146 base-pairs of DNA wrapped around histone proteins, and a linker, which contains between 20 and 100 basepairs of DNA. The physiologically relevant conformation of chromatin is a compact, $30 \mathrm{~nm}$ thick fiber (for a review, see Butler, 1983; Felsenfeld \& McGhee, 1986; Van Holde, 1989; Widom, 1989). These $30 \mathrm{~nm}$ fibers are believed to fold into looped domains imposing a yet higher-order structure on chromatin. The three-dimensional structure of the $30 \mathrm{~nm}$ fiber and the way in which it is further organized have not yet been determined. In fact, our knowledge about the internal structure of the fibers is limited to knowing the diameter, radial density distribution and mass per unit length (Williams et al., 1986; Smith et al., 1990; Williams \& Langmore, unpublished results; Athey et al., 1990).

Here we will focus attention on the relationship between chromatin dynamics and static structure. Many studies have shown that the conformation of chromatin becomes increasingly compact as the cation concentration is increased (see e.g. Thoma et al., 1979; Suau et al, 1979; Widom, 1986). In a low salt medium charge repulsion causes the chromatin to adopt an extended beads-on-a-string conformation. In contrast, at higher cation concentrations the unfavorable electrostatic interactions are reduced and the $30 \mathrm{~nm}$ fiber is favored. Typically, chromatin gradually compacts into the $30 \mathrm{~nm}$ struc ture either as the monovalent salt concentration is raised from 10 to $150 \mathrm{mM}$, or (in the absence of significant monovalent salt) as divalent cations bind to most of the DNA phosphates (Finch \& Klug, 1976; Widom, 1986; Sen \& Crothers, 1986; McGhee et al., 1980, 1983). Furthermore, side-by-side aggregation of the $30 \mathrm{~nm}$ fibers can be induced by multivalent cations (Olins \& Olins, 1972; Langmore \& Paulson, 1983).

Here we present the first study of the effects that salt-induced compaction and aggregation have on the slow dynamics of chromatin in intact nuclei. We have used a relatively novel spectroscopic technique, polarized fluorescence recovery after photobleaching (pFRAPt: Velez \& Axelod, 1988; Scalettar et al., 1988) to monitor reorientational motion, because measurements can be made on a small quantity of a complex biological sample and very slow motions can be studied. We were particularly interested in studying nuclei because nuclear chromatin concentrations are so high (tens to hundreds of $\mathrm{mg} / \mathrm{ml}$ : Rill, 1986; Livolant \& Maestre, 1988) that one might expect interchromatin interactions to suppress the large amplitude (slow) motions of nuclear chromatin. Moreover, important processes such as transcription and replication rely on dynamic events that involve large-scale

$\dagger$ Abbreviations used: pFRAP, polarized fluorescence recovery after photobleaching; PMSF, phenylmethylsulfonyl fluoride; $\mathrm{kb}, 10^{3}$ base-pairs; Etbr, ethidium bromide. unfolding of chromatin molecules; in principle, similar motions can be studied in vivo with the pFRAP technique. Aspects of the salt-induced unfolding of chromatin in nuclei are probably related to important conformational changes found in living cells. For the experiments described here, Necturus erythrocytes were usually used because the cells are transcriptionally quiescent and most of the chromatin highly condensed. Nuclei from 18-hour sea urchin embryos were also studied, because the cells are active in transeription and replication and most of the chromatin was not highly aggregated.

\section{Theory}

\section{(a) Description of the $p F R A P$ iechnique}

The theory of $\mathrm{pFR} \Lambda \mathrm{P}$ has becn described in detail (Velez \& Axelod, 1988; Scalettar et al, 1988). In a pFRAP experiment, fluorescently labeled molecules are exposed to a brief $(\mu \mathrm{s})$, intense pulse of polarized laser light. This pulse of light serves preferentially to bleach (render non-fluorescent) molecules with absorption moments aligned with a significant component parallel to the polarization of the beam during the bleach. If the bleach pulse is much shorter than the characteristic rotation time in the sample, it creates an anisotropic angular distribution of unbleached fluorophore. A much less intense polarized probing beam is then used to excite fluorescence from unbleached dye. The time dependence of the resulting post-bleach fluorescence is determined by the return of the dye to an isotropic angular distribution. The polarization of the bleach pulse alternates between two perpendicular directions, while the polarization of the probe beam is fixed. For one polarization mode (the parallel mode) the bleach and probe polarizations coincide; in the other bleaching polarization (perpendicular mode) they are orthogonal. Typical parallel, $F_{||}(t)$, and perpendicular, $F_{\perp}(t)$, mode $\mathrm{pFRAP}$ data are shown in Figure 1.

In the microsecond time regime the temporal dependences of the parallel and perpendicular mode pFRAP signals are determined by rotational motion and a photophysical recovery of the fluorophore (Velez \& Axelrod, 1988; Scalettar et al., 1988). However, if an anisotropy function, $r_{b}(t)$, is construeted from the pFRAP data obtained in the parallel and perpendicular modes, the time dependence of $r_{\mathrm{b}}(t)$ is determined only by rotational motions, i.e. the anisotropy function is not sensitive to the photophysical recovery of the dye (Velez \& Axelrod, 1988). The anisotropy is defined by:

$r_{\mathbf{b}}(t)=\left[\Delta F_{\|}(t)-\Delta F_{\perp}(t)\right] /\left[\Delta F_{\|}(t)+2 \Delta F_{\perp}(t)\right]$,

where $\Delta F_{\|}(t)=F_{0}-F_{\|}(t)$, i.e. the difference between the pre-bleach fluorescence, $F_{0}$, and the fluorescence at time $t$ in the parallelmode, and $\Delta F_{\perp}(t)=F_{0}-F_{\perp}(t)$

Two important parameters can be extracted from $r_{b}(t)$. They are the initial value of the anisotropy, 


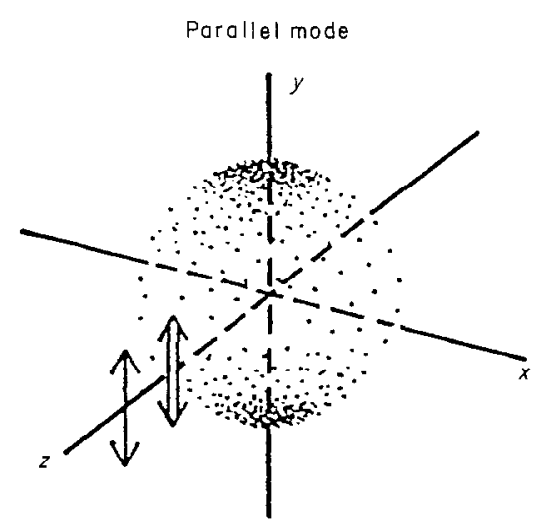

(a)

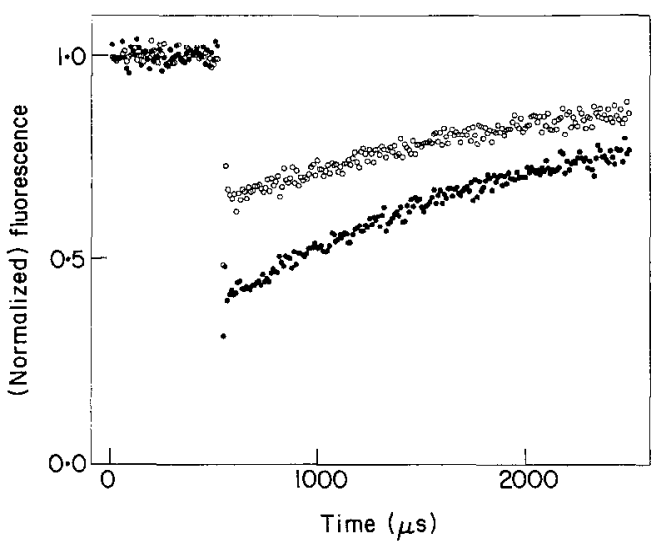

(c)
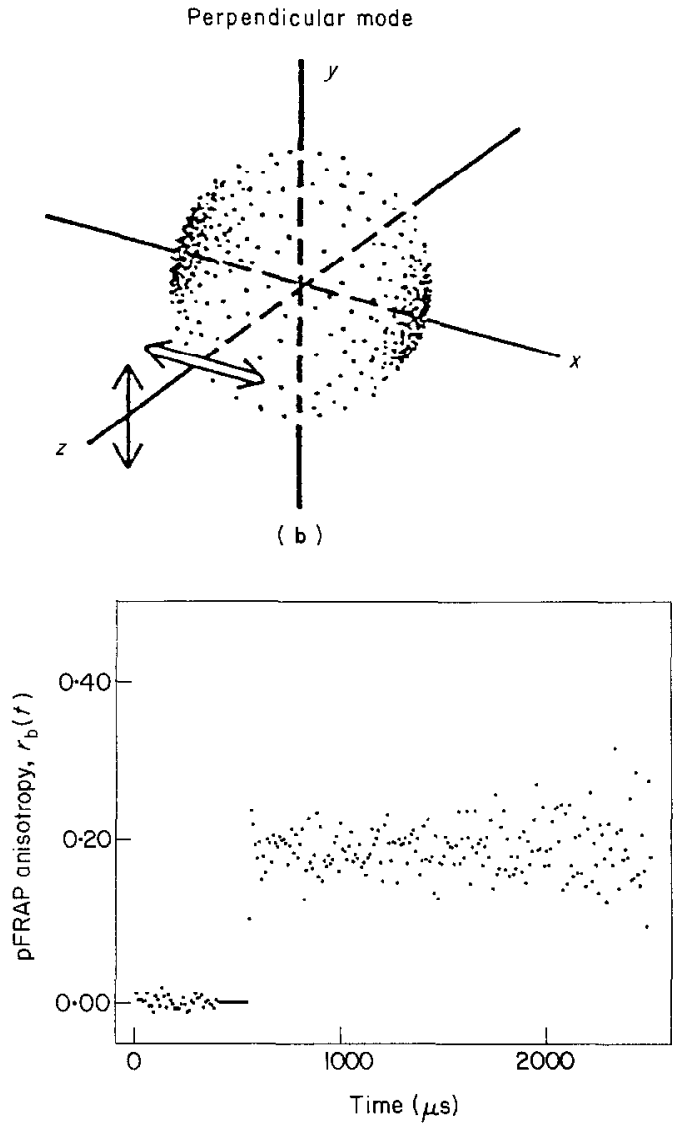

(d)

Figure 1. Schematic representation of the bleaching and probing events that take place in a pFRAP experiment; representative $\mathrm{pFRAP}$ data obtained from dry DNA are also shown. (a) The sample is first illuminated with a brief $(\mu \mathrm{s})$ intense pulse (double arrow) of polarized laser light; this pulse will create a region (darkened area) of preferentially bleached fluorophore around the axis of polarization of the bleach beam. In the parallel mode, a much attenuated (single arrow) probe beam with the same polarization as the bleach beam is then directed onto the sample. The post-bleach fluorescence signal that results is initially small because the probe beam is attempting to excite bleached dye; however, as rotational diffusion leads to an exchange of bleached and unbleached fluorophore the fluorescence will recover at a rate dictated by rotational and photophysical relaxation times. (b) In the perpendicular mode, the polarization of the blcach beam (double arrow) is orthogonal to that of the probe (single arrow) beam and, therefore, initially, the probe beam is not exciting the bleached dye. (c) Thus, if the molecules in the sample are rotating slowly (on the $\mu$ s time-scale), the bleach will appear to be deeper in the parallel mode (the lower curve in (c)) than in the perpendicular (upper curve (c)). Moreover, with time $F_{\|}(t)$ and $F_{\perp}(t)$ will tend toward the same asymptotic value. In contrast, if the molecules in the sample are tumbling rapidly on the $\mu \mathrm{s}$ time-scale, $F_{\|}(t)$ and $F_{\perp}(t)$ will superimpose. $(\mathrm{d})$ The ratio, $r_{\mathrm{b}}(t)=$ $\left[\Delta F_{\|}-\Delta F_{\perp}(t)\right] /\left[\Delta F_{\|}+2 \Delta F_{\perp}\right]$ associated with the data in (c) is shown. The ratio contains the dynamic component of the recovery but not the photophysical component.

$r_{b}(0)$, and the time constant, $\tau$, associated with the rate at which $r_{\mathbf{b}}(t)$ decays to zero. The initial value of the anisotropy gives a measure of the extent of fast $(\leq 10 \mu \mathrm{s})$ motion in the sample. If the fluorophores reorient completely during the bleach pulse (generally equal to $10 \mu \mathrm{s}$ here) there will be no difference between $F_{11}(0)$ and $F_{\perp}(0)$ and $r_{b}(0)$ will be zero. In contrast, if the molecules are immobile during the bleach, $r_{\mathrm{b}}(0)$ should attain its theoretical maximum value, 4/7. Typically, $r_{b}(0)$ lies somewhere between these two extrema, and then we conclude that there is partial relaxation during the bleaching pulse.

The time constant gives a measure of the rate at which slowly rclaxing molecular motion leads to depolarization in the sample. For relatively simple systems the temporal decay of the pHRAP anisotropy can be quantitatively related to specific quantitics, such as rotational diffusion cocfficients. For the complex systems under study here, there is not a simple realistic model for the dynamics from which quantitative conclusions can be derived. However, changes in $r_{b}(0)$ and $\tau$ ean still be related to important features of chromatin dynamics and structure.

\section{(b) Characterization of photophysical relaxation in $p F R \Lambda P$ experiments}

The photophysical recovery time and depth of bleach $\left(\left[F_{0}-F_{\|}(0)\right] / F_{0}\right)$ increase markedly in dcoxygenated samples, suggesting that triplet states 
mediate photophysical recovery (Scalettar et al., 1990). Although the origin of the oxygen sensitivity is not particularly important here, it is important to note that deoxygenation enhances the signal-tonoise ratio in $p F R A P$ by increasing the magnitude of the quantity $\left(F_{0}-F_{\|}(t)\right) / F_{0}$. In the presence of $\mathrm{O}_{2}$, $F_{0}-F_{\|}(t)$ rapidly decreases, reducing the signal-tonoise ratio at long times. For this reason, all the chromatin and nuclei samples studied here were deoxygenated. Control experiments demonstrated that deoxygenation did not affect the value of $r_{\mathrm{b}}(t)$.

\section{Materials and Methods}

\section{(a) Buffers}

Wash buffer consisted of $130 \mathrm{~mm}-\mathrm{NaCl}, 5 \cdot 0 \mathrm{~mm}-\mathrm{KCl}$, $2.0 \mathrm{~mm}-\mathrm{MgCl}_{2}, \quad 10 \mathrm{~mm}-\mathrm{Hepes} \quad(\mathrm{pH} \mathrm{7} \cdot 0), \quad 0 \cdot 1 \mathrm{~m}$-sucrose. Synthetic sea water was prepared from a powder obtained from Instant Ocean (Eastlake, $\mathrm{OH}$ ). Buffer $\mathrm{A}$ contained $60 \mathrm{~mm}-\mathrm{KCl}, \quad 15 \mathrm{~mm}-\mathrm{NaCl}, \quad 15$ mm-Pipes (pH 7.0), $0.5 \mathrm{~mm}$-spermine, $0.5 \mathrm{~mm}$-spermidine, $2.0 \mathrm{~mm}$-EDTA, $0.02 \%(\mathrm{w} / \mathrm{v}) \mathrm{NaN}_{3}$. Buffer mMB consisted of 5 mm-Pipes ( $\mathrm{pH}$ 7.0), $3 \mathrm{~mm}-\mathrm{MgCl}_{2}, 18 \mathrm{~mm}-\mathrm{NaCl}, 72 \mathrm{~mm}-\mathrm{KCl}$; Buffer $\mathrm{mEB}$ consisted of $\mathrm{mMB}$ in which $3 \mathrm{~mm}$-EOTA replaced $\mathrm{Mg}^{2+}$. Micrococeal nuclease digestion buffer contained $60 \mathrm{~mm}-\mathrm{KCl}, \quad 15 \mathrm{~mm}-\mathrm{NaCl}, \quad 15 \mathrm{~mm}-\mathrm{Pipes}(\mathrm{pH} 70)$, $0.5 \mathrm{mM}-\mathrm{CaCl}_{z}, \quad 0.25 \mathrm{~mm}-\mathrm{MgCl}_{2}, \quad 0.1 \mathrm{~mm}$-phenylmethylsulfonyl fluoride (PMSF), and $0.02 \% \mathrm{NaN}_{3}$.

\section{(b) Isolation of nuclei}

Necturus maculosus (mudpuppy) and Strongylocentrotus purpuralus (sea urchin) were obtained from Charles Sullivan (Nashville, TN) and Alacrity Marine Biological Supply (Redondo Beach, CA), respectively. Erythrocytes from Necturus were obtained by heart puncture; sea urchin embryos from S. purpuratus were grown to $18 \mathrm{~h}$ as described by Workman \& Langmore (1985). All subsequent steps were performed at $4^{\circ} \mathrm{C}$, all wash volumes were at least 10 times the volume of the pellet, and each centrifugation of the Necturus samples involved spinning at $60 \mathrm{~g}$ for $6 \mathrm{~min}$.

Necturus erythrocytes were washed twice in wash buffer to separate the cells from lymphocytes. The erythrocytes were then incubated for $l \mathrm{~h}$ on ice in wash buffer with 3 mM-iodoacetate, and $0.2 \mathrm{~mm}$-PMSF to inhibit proteases. The cells were then lysed by washing 3 times in buffer A with $0.1 \%(\mathrm{w} / \mathrm{v})$ digitonin, 1 mM-iodoacetate, and $0.1 \mathrm{~mm}-\mathrm{PMSF}$, and three times in buffer A with $0.1 \%$ Non-idet P40, $1 \mathrm{mu}$-iodoacetate, and 0.1 mu-PMSF. At this point the cells had lysed and were free from cellular debris. Nuclei (at $1 \mathrm{mg} \mathrm{DNA} / \mathrm{ml}$ ) were quickly frozen in $50 \%$ glycerol/buffer A by placing tubes into methanol cooled with solid $\mathrm{CO}_{2}$. The nuclei were stored at $-70^{\circ} \mathrm{C}$. Before nuclei were used in a pFRAP experiment, they were washed 3 times in mMB buffer and then stored at $4^{\circ} \mathrm{C}$ in mMB or mEB buffer.

Sea urehin nuclei were isolated from 18-h embryos as deseribed by Workman \& Langmore (1985). The published method was modified to keep the nuclei in buffer $\mathrm{A}$, including storage in $50 \%$ glycerol at $-70^{\circ} \mathrm{C}$.

\section{(c) Isolation of chromatin fibers}

For the study of isolated chromatin fibers, nuclei $(1 \mathrm{mg}$ in $1 \mathrm{ml}$ ) were pelleted at $4^{\circ} \mathrm{C}$ and washed with $\mathrm{mMB}$ buffer (this procedure removes multivalent cations) and then pelleted again and washed with micrococeal nuclease digestion buffer. Nuclei were then resuspended in $1 \mathrm{ml}$ of micrococcal nuclease digestion buffer and incubated at $22^{\circ} \mathrm{C}$ for $5 \mathrm{~min}$. Nuclei were digested for $5 \mathrm{~min}$ by adding 20 units of micrococcal nuclease (Worthington, Freehold, NJ). Digestion was stopped by increasing the EGTA and $\mathrm{MgCl}_{2}$ to $5.0 \mathrm{~mm}$ and $1.0 \mathrm{~mm}$, respectively. Nuclei were then gently pelleted and resuspended in $1 \mathrm{ml}$ of 0.2 mM-EDTA (pH 7.0), 0.1 mM-PMSF, $0.02 \% \mathrm{NaN}_{3}$ to solubilize the chromatin. Nuclei were allowed to lyse at $4^{\circ} \mathrm{C}$ for $45 \mathrm{~min}$ and were then spun for $5 \mathrm{~min}$ in an Eppendorf centrifuge to remove nuclear debris. Chromatin solutions were brought to physiological ionic strength by adding 0.1 vol. $10 \times \mathrm{mEB}$ buffer. This isolation procedure produces chromatin with the native amount of histone $\mathrm{Hl}$ (S. P. Williams \& J. P. Langmore, unpublished results). The final concentration of the fibers in solution was $\approx 150 \mu \mathrm{g} / \mathrm{ml}$.

Successful isolation of $30 \mathrm{~nm}$ fibers was confirmed by examining the digested fibers in an electron microscope by the method deseribed by Williams et al. (1986). The approximate molecular weight was determined by electrophoresis in $0.3 \%(\mathrm{w} / \mathrm{v})$ agarose. The mode of the molecular weight distribution of soluble chromatin was $55 \mathrm{~kb}$; the weight average molecular weight was $45 \mathrm{~kb}$.

\section{(d) Deoxygenation of samples}

After the nuclei and fibers were isolated, all sample manipulations were conducted in a nitrogen-saturated glove bay. Buffers were deoxygenated by bubbling nitrogen through them for approximately $15 \mathrm{~min}$. Air-tight sample chambers were constructed from $500 \mu \mathrm{l}$ Eppendorf tubes that were slit (in the transverse direction) near the tip with a razor blade; the slit end was then glued to a silanized quartz coverslip with epoxy resin.

\section{(e) Preparation of fluorescently labeled chromatin samples}

Typically $0.1 \mathrm{mg}$ of nuclei was placed into the Eppendorf holder. Ethidium bromide (Etbr) $(2 \mu \mathrm{g} / \mathrm{ml}$ in water) (Sigma Chemicals, St Louis, MO) was then added and assumed to bind stoichiometrically; labeling levels were kept lower than 1 Etbr molecule bound/1000 basepairs. At these low labeling levels energy transfer between ethidium molecules is negligible (Ashikawa el al., 1983). The total sample volume was increased to $400 \mu l$ by adding $\mathrm{mMB}$ or $\mathrm{mEB}$ buffer. We waited about $30 \mathrm{~min}$ for the nuclei to settle onto the quartz coverslip and for the ethidium to stain the chromatin. After this time, the fluorescence was confined to regions containing chromatin, and the nuclei essentially formed a continuous monolayer on the coverslip. Thus, as the microscope stage was translated (see section ( $g$ ), below) the light remained focussed on nuclei. The data were not affected by the fact that the beam occassionally passed over an area with no nuclei because the fluorescence count rate then fell by 2 orders of magnitude. The data also were not affected by the fact that ethidium bromide binds only transiently to the DNA. The average time that an ethidium molecule remains bound to $\mathrm{DNA}$, once it has intercalated, is the inverse of its off rate, or about $50 \mathrm{~ms}$ (Magde et al., 1974). Thus bound ethidium does not come off DNA on the timescale $(\leq 2 \mathrm{~ms}$ ) over which we have monitored internal motion. Moreover, if a significant fraction of the ethidium had come off the chromatin during the post-bleach period, we would have seen a very rapid recovery associated with the independent ethidium motion. 


\section{(f) Manipulation of salt concentration}

Salt concentrations were manipulated by serial dilution of the monovalent salt or chelation of magnesium with EDTA. Specifically, mMB buffer was converted to $\mathrm{mEB}$ buffer by adding enough EDTA to chelate the $\mathrm{Mg}^{2+}$ in the $\mathrm{mMB}$ buffer and to raise the free EDTA concentrations to $3 \mathrm{~mm}$. The monovalent salt concentration was manipulated by removing an appropriate volume of buffer from the top of the sample and replacing it with an equal volume of 5 mm-Pipes, 3 mm-EDTA. The sample was then gently pipetted and incubated $30 \mathrm{~min}$ before beginning the next experiment to ensure that the salt distribution in the solution was uniform. 'The binding constant of ethidium bromide is a function of salt concentration; however, our calculations (and the fact that the total fluorescence count rate did not vary as the salt concentration was changed) indicate that the number of Etbr molecules bound per nucleus did not change significantly during the salt studies. The expected decrease in binding with increasing salt concentration would produce effects opposite to those reported here.

\section{(g) Description of apparatus and pFRAP experimental methods}

The pFRAP apparatus used was very similar to that described (Velez \& Axelrod, 1988). After each round of bleach and probe, the stage was moved horizontally about one beam spot diameter $(\approx 3 \mu \mathrm{m})$ by a computercontrolled stepping motor. In this way it was possible to obtain data from about $10^{5}$ areas without bleaching the same nucleus twice. A microscope was used to collect and focus light because effective bleaching can be achieved with focussed light and effective detection can be achieved with a high numerical aperture lens.

For all the chromatin experiments we used a $10 \mu \mathrm{s}$ bleaching pulse. The pre-bleach and post-bleach fluorescence count rates were monitored for 50 and 200 time points $(10 \mu \mathrm{s} /$ point $)$, respectively. Typically the prebleach fluorescence signals yielded $\sim 100,000 \mathrm{cts} / \mathrm{s}$. About $2 \mathrm{~h}$ of signal averaging were usually required to obtain a signal-to-noise ratio of about $20 / 1$.

The anisotropy obtained from a pFRAP experiment is a function of the amount of bleaching induced in the sample (Velez \& Axelrod, 1988). The anisotropy will decrease if the depth of bleach is too large. Hence, we kept the bleach modest $(25 \%$ to $40 \%)$; at such bleaching levels $r_{\mathrm{b}}(t)$ is essentially bleach-independent. The desired amount of bleaching was obtained from ethidium-stained samples by adjusting the laser power output to $2 \mathrm{~W}$, when using the $514.5 \mathrm{~nm}$ line of a Coherent $15 \mathrm{~W}$ argon ion laser, and then attcnuating the beam with an optical density $0 \cdot 6$ filter. Since there is considerable loss of light in the optical components as the beam travels from the laser to the microscope, only about $100 \mathrm{~mW}$ of power actually impinges on the sample during the bleach pulse. We used a $10 \times$ glycerine immersion objective with a numerical aperture of 0.5 to focus the light onto the sample. These experimental conditions lead to negligible light-associated temperature rises in our samples (Velez \& Axelrod, 1988; Scalettar et al., 1988).

\section{(h) Data analysis}

Time constants and initial values of the anisotropy, $r_{\mathrm{b}}(t)$, were determined by fitting the raw data to a single decaying exponential function. Although there is no $a$ priori reason for $r_{\mathrm{b}}(t)$ to exhibit an exponcntial decay, it was found empirically that a single exponential fit the data quite well. The algorithm utilized by Curfit (Bevington, 1969) was adapted for use in our curvefitting program. All data points were used to determine the bestfit parameters, but the weighting attached to each time point was set by its statistical significance. When necessary, data were also adjusted for slight differences in the intensities of parallel and perpendicular polarized light.

\section{Results}

(a) Diffusion of chromatin in mudpuppy nuclei at physiological salt concentration

The reorientational motion of native chromatin fibers in intact mudpuppy nuclei was measured in near physiological (mMB buffer) salt. The polarized photobleaching data obtained from mudpuppy nuclei suspended in mMB buffer are displayed in Figure 2(a). The substantial difference between the

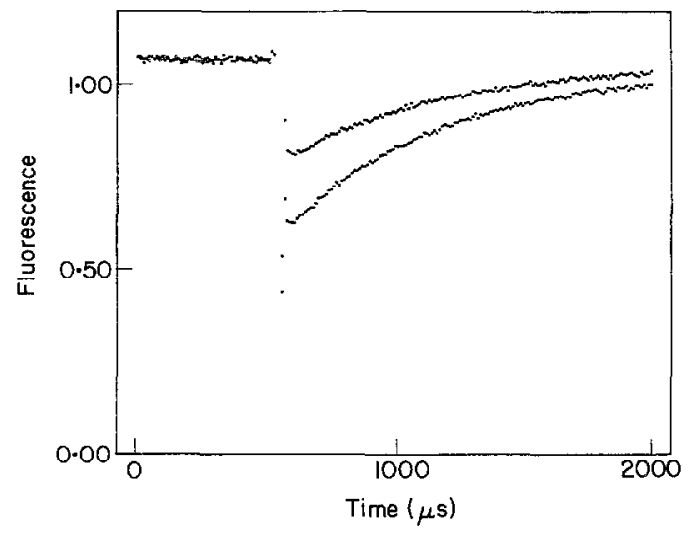

(a)

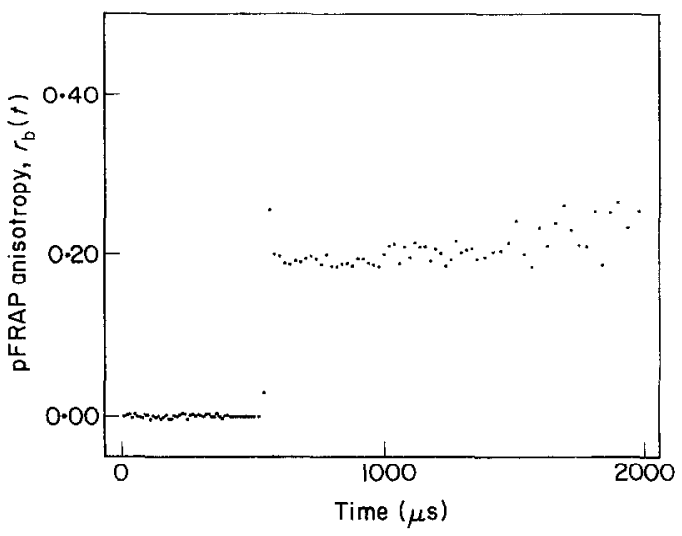

(b)

Figure 2. (a) Polarized photobleaching recovery curves, $F_{\sharp}(t)$ (lower) and $F_{\perp}(t)$ (upper) obtained from a sample of mudpuppy nuclei suspended in mMB buffer and $(b)$ the associated anisotropy. The temporal behavior of the individual recovery curves does not convey the impression that this aggregated chromatin sample is rotationally immobile; this fact becomes apparent only upon examination of the anisotropy function. Note that although the data were collected at $10 \mu \mathrm{s}$ sample intervals (bins), the anisotropy functions in this and Figs 3 and 6 were obtained by adding together neighboring bins (for the long time data points only); this procedure improves the signal-to-noise ratio at long times. 


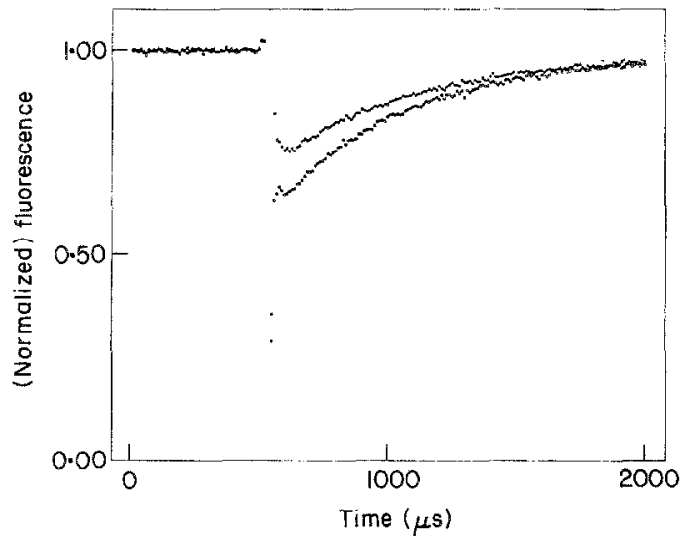

(a)

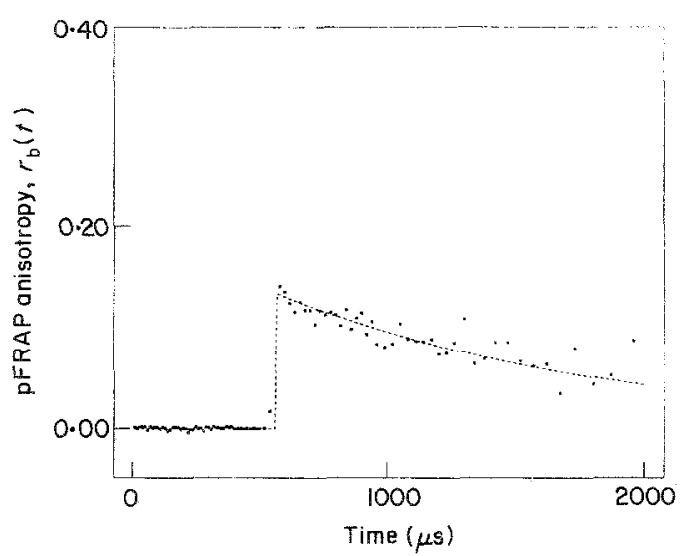

(b)

Figure 3. (a) Individual recovery curves and (b) anisotropy function obtained from mudpuppy nuclei suspended in mEB buffer. Comparison with the data of Fig. 2(b) shows that chelation of magnesium has converted an immobile sample inte a mobile one. It is also elear, from the raw data, that the initial anisotropy decreased when the $\mathrm{Mg}^{2+}$ was removed.

bleach depth in the parallel mode and in the perpendicular mode shows that this sample retains a substantial amount of anisotropy at the end of the ten microsecond bleaching pulse (see the legend to Fig. 1). It is also evident, upon comparing $F_{\|}(t)$ and $F_{\perp}(t)$, that the anisotropy is quite long-lived, i.e. reorientation in this sample is slow. These statements can be made quantitative by calculating $r_{\mathrm{b}}(t)$. It is then seen (see Fig. $2(b)$ ) that the initial value of the anisotropy, $0 \cdot 20$, is positive and quite large. Moreover, $r_{\mathrm{b}}(t)$ is basically flat for several milliseconds after bleaching. An estimated lower bound for the time constant $(40 \mathrm{~ms})$ was obtained by assuming that there is less than a $5 \%$ decay of anisotropy in two milliseconds and that an exponential deseribes the temporal dependence of $r_{\mathrm{b}}(t)$.

\section{(b) Diffusion and compaction of chromatin in mudpuppy nuclei as a function of salt concentration}

The reorientational motion of native chromatin in mudpuppy nuclei was also followed as a function of salt concentration. pFRAP data were obtained from mudpuppy samples that contained no divalent cations and had monovalent salt concentrations of $90 \mathrm{mM}$ (mEB), $69 \mathrm{~mm}, 54 \mathrm{mM}, 45 \mathrm{mM}, 36 \mathrm{mM}$, $22 \mathrm{mM}$ and $11 \mathrm{~mm}$-Pipes-EDTA buffer. $F_{\|}(t), F_{\perp}(t)$ and $r_{\mathrm{b}}(t)$ are shown for mudpuppy nuclei in $\mathrm{mEB}$ buffer in Figure 3. Figures 4 and 5 are summaries of the best-fit initial anisotropies and time constants for all of the salt concentrations measured. Both the initial anisotropy and the relaxation time are monotonically increasing functions of salt concentration. Comparison of the pFRAP data obtained from nuclei in physiological salt and in $\mathrm{mEB}$ buffer shows that simple chelation of the $\mathrm{Mg}^{2+}$ converts a rotationally immobile sample into one with a correlation time of 1.2 milliseconds. In $11 \mathrm{~mm}$-monovalent salt, the chromatin is so mobile that the sample is completely depolarized before the bleach period has ended, i.e. $r_{b}(0)=0$. These salt-induced changes in relaxation rate were reversible. Addition of monovalent or divalent cations to low-salt nuclei resulted in the return of anisotropy.

Several interesting regions of transition can be identified in Figures 4 and 5. Between 11 and $50 \mathrm{~mm}$-monovalent salt there is a steep increase in $r_{b}(0)$, accompanied by a gradual increase in $\tau$. Apparently compaction is dramatically inhibiting the extent of rapidly relaxing motion. From 60 to 90 mu-monovalent salt $r_{b}(0)$ is essentially constant, but the time constant increases dramatically. Thus, as salt changes refine the structure of the $30 \mathrm{~nm}$ fibers the increase in rigidity is primarily manifest in

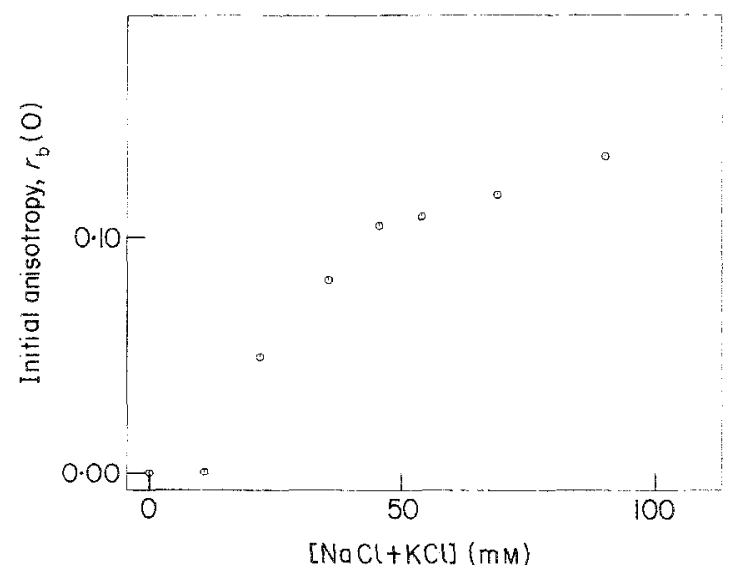

Figure 4. Best-fit initial anisotropies obtained from mudpuppy nuclei suspended in solutions of various salt concentrations. The anisotropy is plotted as a function of the $\mathrm{NaCl}+\mathrm{KCl}$ concentration (mM). All buffers contained 5 mM-Pipes ( $\mathrm{pH} 7), 3$ mM-CDTA, but they differed in their total $\mathrm{NaCl}+\mathrm{KCl}$ concentration. The $\mathrm{NaCl} / \mathrm{KCl}$ concentrations were: $\quad 72 \mathrm{~mm}-\mathrm{KCl}, \quad 18 \mathrm{~mm}-\mathrm{NaCl}$; $55 \mathrm{~mm}-\mathrm{KCl}, \quad 14 \mathrm{~mm}-\mathrm{NaCl} ; \quad 43 \mathrm{~mm}-\mathrm{KCl}, \quad 11 \mathrm{~mm}-\mathrm{NaCl}$; $36 \mathrm{~mm}-\mathrm{KCl}, \quad 9 \mathrm{~mm}-\mathrm{NaCl} ; \quad 29 \mathrm{~mm}-\mathrm{KCl}, \quad 7 \mathrm{~mm}-\mathrm{NaCl}$; $18 \mathrm{~mm}-\mathrm{KCl}, 4 \mathrm{~mm}-\mathrm{NaCl} ; 9 \mathrm{~mm}-\mathrm{KCl}, 2 \mathrm{~mm}-\mathrm{NaCl}$. The magnesium containing (mMB buffer) solution gave the highest initial anisotropy, $0 \cdot 19$; this point is not plotted above. 


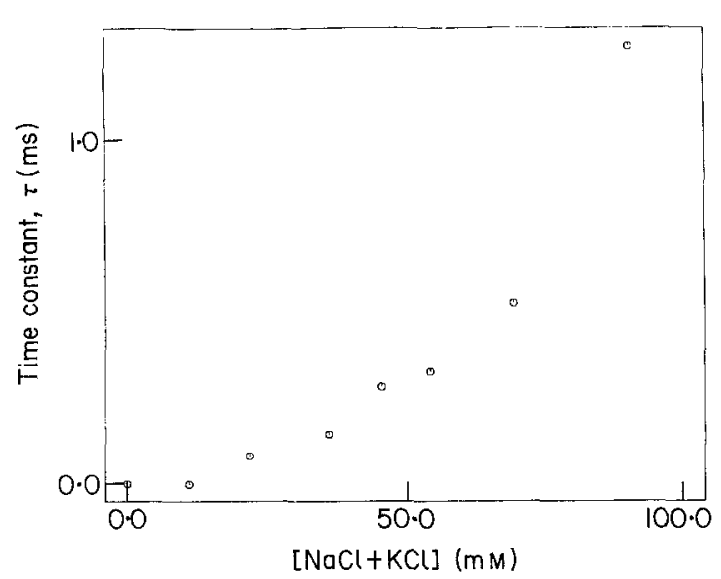

Figure 5. Best-fit relaxation times associated with the anisotropies shown in Fig. 4. The mMB point is not plotted because of its long (nominally infinite) relaxation time.

the rate of decay of the slow large amplitude motions of chromatin. The initial anisotropy and time constant both increase markedly upon addition of $\mathrm{Mg}^{2+}$, demonstrating that aggregation inhibits a broad temporal spectrum of chromatin motion.

\section{(c) Diffusion of chromatin in sea urchin nuclei}

We have compared the rotational mobility of chromatin fibers in sea urchin and mudpuppy nuclei at physiological salt concentrations (see Fig. 6). In both types of nuclei the chromatin is highly immobile; however, the sea urchin samples exhibited about $25 \%$ less anisotropy than the mudpuppy samples. The best estimate for $\tau$ was seven milliseconds, showing that the chromatin of the more active nuclei reoriented a minimum of six times faster than the chromatin from the inactive Necturus cells.

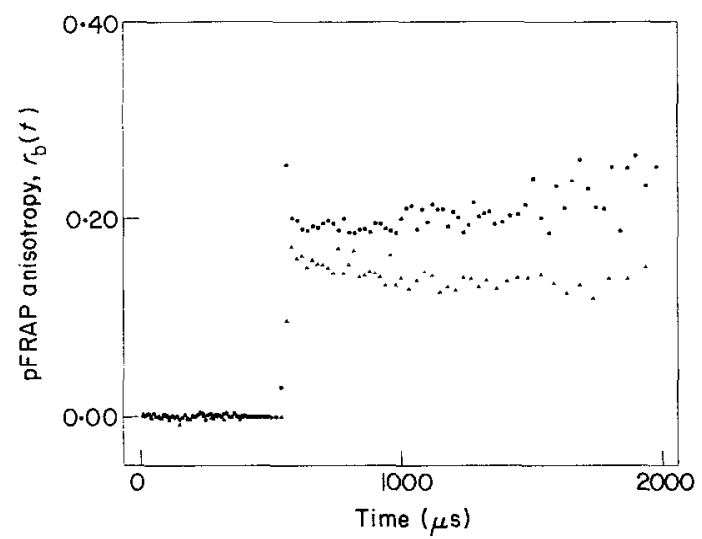

Figure 6. Comparison of the dynamics of sea urchin (A) and mudpuppy (O) nuclear chromatin in $\mathrm{mMB}$ buffer. The initial value of the anisotropy and the time constant are both smaller in the sea urchin sample.

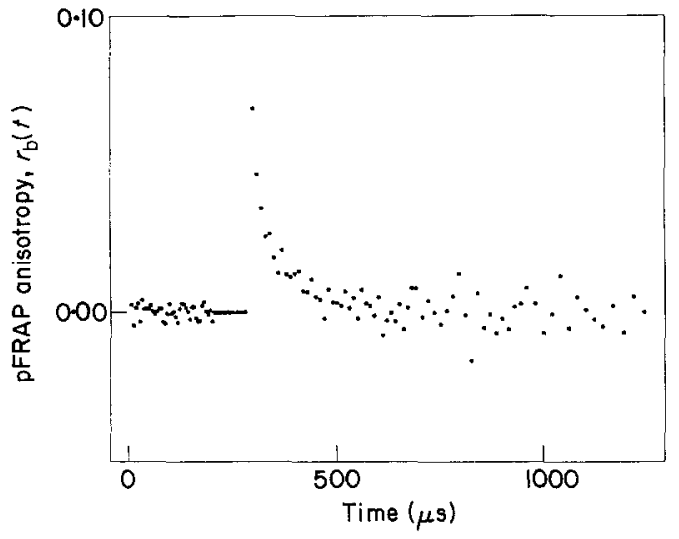

Figure 7. Anisotropy function obtained from digested mudpuppy chromatin fragments $(150 \mu \mathrm{g} / \mathrm{ml})$ in $\mathrm{mEB}$ buffer. This sample contained chromatin fragments that were broadly distributed in molecular weight.

We also attempted to study the salt dependence of chromatin dynamies in sea urchin nuclei. The mobility of sea urchin chromatin in nuclei again increased as the salt concentration was lowered. However, the sea urchin nuclei lysed when the salt concentration was decreased to about $50 \mathrm{~mm}$, causing irreversible changes.

\section{(d) Diffusion of digested chromatin fibers in solution and nuclei}

We also measured the reorientational dynamies of nuclease-digested chromatin fibers in solution and in nuclei. The anisotropy obtained from a $150 \mu \mathrm{g} / \mathrm{ml}$ solution of digested chromatin $\left(M_{\mathrm{w}}=45 \mathrm{~kb}\right)$ in $\mathrm{mEB}$ buffer is shown in Figure 7. The initial anisotropy is 0.05 and $r_{\mathrm{b}}(t)$ decays with a time constant of 100 microseconds. Most of the digested fibers were not solubilized but remained in the nucleus. The nuclear-bound fragments have a slightly larger weight distribution $\left(M_{\mathrm{w}}=53 \mathrm{~kb}\right)$ than those released into solution. The nuclear-bound fibers were much more immobile $\left(r_{\mathrm{b}}(0) \approx 0 \cdot 14 ; \tau \approx 2 \mathrm{~ms}\right)$ than their soluble analogues. Thus, cleavage per se does not lead to increased mobility, but solubilization does. Attachment of the insoluble chromatin to the nuclear matrix or the high concentration of the molecules left within the nucleus seems to be critical for immobilization.

\section{Discussion}

(a) Reorientation of chromatin in nuclei

Because the focus here is on the dynamics of nuclear chromatin, we briefly discuss the spectrum of motions that chromatin is likely to exhibit and how this motion is manifest in a pFRAP experiment. Neither the static nor the dynamic flexibility of chromatin has been well studied. Previous measurements of dynamic flexibility have been limited to the very rapidly (ns) relaxing motions 
(Shindo et al., 1980; Klevan et al., 1979). Such studies have shown that the ethidium fluorophore wobbles when bound to DNA (Magde et al., 1983) and that core and linker DNA twist on timescales shorter than approximately 100 nanoseconds (Wang et al., 1982; Schurr \& Schurr 1985; Ashikawa et al., 1983, 1985; Hurley et al., 1982; Hard et al., 1988). These rapid components of the dynamics cause the initial value of the pFRAP anisotropy to be smaller than its static limiting value, $4 / 7$.

Here we have extended previous studies of dynamic flexibility by systematically monitoring the microsecond and millisecond internal motions of nuclear chromatin and by relating these slow dynamics to chromatin static structure. In this long time domain it is probable that independent rotations of internal segments and lateral deformations of chromatin give rise to the anisotropy decay. Other sources of microsecond-millisecond reorientation certainly may exist; we cite the above two mechanisms explicitly as examples because both have been demonstrated for naked DNA (Ding et al., 1972; Schmitz \& Schurr, 1973).

Another possible mechanism for motion, rigid body rotation of an entire nuclear chromosome, is unlikely to be the origin of the millisecond relaxation detected here. The calculated relaxation time for an entire mudpuppy chromosome containing $\geq 10^{9}$ base-pairs lies far outside the time domain probed here. However, millisecond relaxation could represent independent rigid rotalion of topologically independent units of chromatin. Paulson \& Laemmli (1977) estimated a domain to be 30 to $90 \mathrm{~kb}$ in size for HeLa tissue culture cells. Urodeles such as Necturus are estimated to have domains more than ten times larger than those for human (HeLa) cells (MacGregor, 1980). If potential interactions with the nuclear matrix are neglected and a topological unit is assumed to fold back onto itself to form a freely diffusing rigid rod containing $500 \mathrm{~kb}$ (length about $2 \mu \mathrm{m}$; Williams et al., 1986), the calculated long axis diffusion coefficient, $D_{\text {ll }}$, is $336 \mathrm{~s}^{-1}$ (Broersma, 1960); $\tau_{\|}$is of the order $1 / D_{\|}$or three milliseconds.

\section{(b) Salt dependence of chromatin reorientation}

Salt-induced transitions of chromatin conformation and aggregation provide a model system with which to study the compaction and aggregation processes that occur during transcription, mitosis and replication. We have found that the internal dynamics of chromatin mirrors the salt dependence of the internal structure and side-by-side aggregation of chromatin. Thus, we can study the cooperativity of the condensation of chromatin, the flexibility of the various conformers of chromatin and the relative effectiveness of a given cation in producing condensation. The advantage of the pFRAP method is that dynamic measurements can be made when the chromatin is still present in the nucleus or, in principle, while in a living cell.
Studies of chromatin structure have shown that at physiological salt concentrations (high monovalent salt and in the presence of $\mathrm{Mg}^{2+}$ ) chromatin exists as a compact $30 \mathrm{~nm}$ fiber and that these fibers are packed together side-by-side (aggregated). The pFRAP data reported here show that the motion of the linker region is very restricted under these conditions. (Our signal is expected to. reflect primarily linker dynamics because it has been shown that ethidium binds preferentially to this region of chromatin if the level of dye labeling is kept low (Paoletti et al., 1977; Erard et al., 1979 Genest et al., 1981).) Specifically, we find that in physiological buffers the pFRAP anisotropy is constant and large $\left(r_{b}=0 \cdot 2\right)$; this result indicates that the linker DNA is immobilized on time scales that range between 10 microseconds and 2 milliseconds. However, our data also show that fluorophores bound to aggregated fibers retain a substantial amount of localized mobility; these local, rapidly relaxing motions cause deviation of $r_{\mathrm{b}}(0)$ from the rigid limit, $4 / 7$. This "fast" depolarization seen in the pFRAP experiments is probably due to a combination of dye wobble and twisting motions. Such rapid relaxation is ubiquitious; even in immobilized (dry) DNA samples the initial anisotropy is only $0 \cdot 2$ (see Fig. $1(d)$ ).

Electron microscopy and X-ray studies have shown that when $\mathrm{Mg}^{2+}$ is removed the $30 \mathrm{~nm}$ fiber is intact but the chromatin is dispersed (see e.g. Thoma et al., 1979; Langmore \& Paulson, 1983). Under these conditions we see in the optical microscope that the chromatin becomes uniformly dispersed throughout the nucleus. The initial pFRAP anisotropy decreases to 0.14 and $r_{b}(t)$ decays characteristically to zero in about one millisecond. This millisecond relaxation may arise from slow lateral deformations of the $30 \mathrm{~nm}$ fiber that were suppressed when the chromatin was aggregated. Alternatively: as demonstrated in section (a), above, this relaxation can also be attributed to a long axis rigid rod rotation of the topologically independent units that have been postulated to exist in nuclear chromatin (for a review, see Nelson et al., 1986).

Upon dilution of the monovalent salt (and in the absence of $\mathrm{Mg}^{2+}$ ) structural studies show that the compact $30 \mathrm{~nm}$ fiber opens to a beads-on-a-string $(10 \mathrm{~nm})$ conformation consisting of loosely packed nucleosome core particles connected by linker DNA. Upon reduction of monovalent salt the pFRAP initial anisotropy and the relaxation time monotonically decrease. Thus, as chromatin unfolds the extent of rapid internal reorientation increases, and the observable slow motions relax more rapidly. We attribute these changes to a gradual unfolding of the $30 \mathrm{~nm}$ conformer, which leads to greater mobility of the linker DNA region. At the lowest salt concentration studied here, we find that the postbleach anisotropy falls to zero; thus in the low salt structure the linker DNA is relatively free to move. In fact, the low salt conformer is so flexible that its dynamics is too rapid to be measured by pERAP. 
For in vitro work it is important to identify the salt conditions that stabilize the physiologically relevant conformation of chromatin. Unfortunately, as Widom (1989) has noted, some experimental data suggest that there is a well-defined endpoint to the structural transitions of chromatin while other data suggest that no such endpoint exists. Here we have found that, when measurements are made on intact nuclei, the pFRAP time constant, $\tau$, continues to change as the salt concentration is varied between 11 and 90 mu-monovalent salt. In contrast, our measurements of $r_{\mathrm{b}}(0)$ as a function of salt concentration do show a semi-plateau from 50 to $90 \mathrm{~mm}$-monovalent salt. Thus, the salt dependence of the larger amplitude internal dynamics of chromatin (the $\tau$ data) indicates that there is no subphysiological endpoint to dynamic/structural change, while the salt dependence of the smaller amplitude internal motion (the $r_{\mathrm{b}}(0)$ data) indicates that a sub-physiological endpoint may exist. To reconcile these two results we note that the saltinduced folding of chromatin might first damp the most rapid internal dynamics of the molecules and might damp the slower internal dynamies at higher salt concentrations. Overall, the pFRAP data indicate gradual structural change without a clearly delineated endpoint.

The pFRAP data both complement and confirm results obtained in a recent fluorescence depolarization study of the dynamics of ethidium bromidestained linker DNA in nuclei (Ashikawa et al., 1985). The fluorescence depolarization experiments indicate that fast (ns) motions (presumably twisting) are inhibited in intact $30 \mathrm{~nm}$ nuclear chromatin fibers. Our data show a similar suppression of linker DNA dynamies upon $30 \mathrm{~nm}$ fiber formation, although, presumably we are seeing the same effect manifest by the slow motions of DNA.

Linear and saturation transfer electron paramagnetic resonance have also been used to examine the dynamics of nuclear chromatin and isolated chromatin fibers (Hurley et al., 1982). It was found that a spin-labeled ethidium bromide intercalated in the linker region of chromatin fibers or nuclear chromatin is less mobile than a spin label intercalated into naked DNA. Hurley et al. report a 100 microsecond rotational correlation time for nuclei in $5 \mathrm{mM}-\mathrm{MgCl}_{2}, 20 \mathrm{~mm}-\mathrm{Tris} \cdot \mathrm{HCl}$ ( $\mathrm{pH} 7 \cdot 5$ ), 250 m.M-sucrose, but state that this figure may be too small because it is difficult to extract long-time dynamical data from computer simulations of electron paramagnetic resonance spectra. Because Hurley et al. have used a viscous buffer and different ionic conditions it is somewhat difficult to compare their result with ours.

\section{(c) Correlation with studies of chromatin structure}

Electron microscopy has shown that all of the chromatin fibers are extensively unfolded below about 15 mm-monovalent salt (Thoma et al., 1979). Here we find that at $11 \mathrm{~mm}$-monovalent salt chromatin exists in a very flexible conformation characterized by zero post-bleach anisotropy. Between 11 and $50 \mathrm{~mm}$-monovalent salt the $r_{\mathrm{h}}(0)$ value increases rapidly and there is a gradual increase in $\tau$. These data indicate that a substantial fraction of the chromatin is becoming less flexible, but the compaction is not yet complete. This observation is consistent with electron microscopy, which shows a disappearance of the beads-on-astring structure and the formation of rather loose, disordered $30 \mathrm{~nm}$ fibers (Thoma et al., 1979). The sedimentation coefficient of chromatin also increases abruptly as the monovalent salt concentration approaches $50 \mathrm{~mm}$ (Butler \& Thomas, 1980). From 50 to $90 \mathrm{~mm}$-monovalent salt the extent of rapid relaxation is nearly constant, while the time constant increases dramatically. Electron microscopy has shown a tightening of the structure in this salt range (Thoma et al., 1979). When $\mathrm{Mg}^{2+}$ is added (at 90 mu-monovalent salt) $r_{\mathrm{b}}(0)$ and $\tau$ increase quickly. It is in this ionic range that the chromosome fibers become less soluble (i.e. aggregate) with few, if any, changes in internal structural (Langmore \& Paulson, 1983). Thus, it is reasonable to attribute the first pFRAP salt transition to packing of the nucleosomes, and the second transition to aggregation of the fibers.

\section{(d) Concentration dependence of chromatin dynamics}

The dynamics of DNA in dilute solution has been the subject of extensive experimental study (for reviews, see Hagerman, 1988; Shibata et al., 1985; Frank-Kamenetskii, 1981). However, in the nuclei of eukaryotic cells, in the nucleoli of prokaryotic cells and in bacterial phage heads, the concentration of chromatin/DNA is enormously high (Kellenberger, 1987; Livolanl \& Maeslre, 1988); therefore, it is also of interest to monitor the dynamics of nucleic asids in concentrated solution and to see if interparticle interactions modify the motions exhibited by these molecules in vivo.

Interaction-induced modification of the translational motion of nucleic acids is a moderately wellstudied phenomenon. (See among others Sorlie \& Pecora (1988) and Scalettar et al. (1989), and references cited therein.) However, the effects that interactions have on the reorientational motion of nucleic acids are much less well characterized. Rill et al. (1983) conducted ${ }^{31} \mathrm{P}$ and ${ }^{13} \mathrm{C}$ nuclear magnetic resonance studies of concentrated, liquid crystalline phases of short DNA molecules and found that at critical DNA concentrations the local (ns) motions of DNA that are monitored by nuclear magnetic resonance are not present. For DNA of several hundred base-pairs in length these critical concenlrations are of the order of $100 \mathrm{mg} / \mathrm{ml}$.

Interaction-dependent modification of the reorientational motion of DNA has also been detected, by Ashikawa et al. (1984), in the bacteriophage head. DNA in phage heads is believed to be ordered in a closely packed structure and, therefore, the phage is an in vivo system in which one might 
expect to see marked inhibition of the mobility of DNA. Ashikawa et al. have detected such inhibition of DNA mobility in wild-type and mutant (DNAdeficient) $\lambda$ phage, and they have correlated dynamie changes with structural changes measured by $\mathrm{X}$-ray scattering. On the timescales ( $\leq 100 \mathrm{~ns}$ ) accessible to a fluorescence depolarization experiment the DNA in both phages was much less mobile than DNA in dilute solution. Moreover, the DNA in wild-type phage was found to be more immobile than the DNA in the mutant (DNA-deficient) phage. Thus, these data indicate that in vivo DNA concentrations are an important determinant of the dynamics of DNA.

Here we have also shown that at physiological salt (mMB buffer) concentrations the ( $\mu$ s and $\mathrm{ms}$ ) internal motions of chromatin are largely inhibited by intermolecular interactions (see Fig. 2). The cxtent to which nuclear chromatin is immobilized at physiological salt concentrations can perhaps best be appreciated by noting that the pFRAP anisotropy obtained from nuclear chromatin fibers in $\mathrm{mMB}$ buffer is the same as is obtained from DNA that is immobilized by drying it down onto a quartz coverslip (see Fig. 1(d)). We attribute the rotational immobilization of nuclear chromatin to fiber aggregation for the following reason. We find that nuclear chromatin in $\mathrm{mMB}$ buffer appears to be rotationally immobile (Fig. 2), while chromatin in $\mathrm{mEB}$ buffer has a one millisecond relaxation time (Fig. 3 ). Since $\mathrm{Mg}^{2+}$ in mMB buffer is believed to induce aggregation of chromatin (which we could see in the microscope) but not to alter its monomeric structure, the change in time constant should reflect a local change in chromatin concentration. The observed increase in $\tau$ indicates that intermolecular aggregation suppresses intramolecular motion.

It is also worth noting that the $45 \mathrm{~kb}$ digested chromatin fibers are much more mobile when suspended in solution at low concentration $(150 \mu \mathrm{g} / \mathrm{ml})$ than when retained at high concentration in the nucleus. This effect may arise from concentration-induced damping of motion or interaction of the chromatin with the nuclear membrane.

\section{(e) Comparison of digested chromatin and naked $D N A$}

We have also compared the microsecond reorientational molion of solubilized $30 \mathrm{~nm}$ chromatin fibers of relatively well-defined length $(\sim 45 \mathrm{~kb})$ to that of a $50 \mathrm{~kb}$ naked DNA from phage $\lambda$ (data not shown). The concentration of DNA in both of the samples studied was $150 \mu \mathrm{g} / \mathrm{ml}$. In such a relatively dilute solution, a sample of naked DNA exhibits essentially no anisotropy at the end of a ten microsecond bleaching pulsc. In contrast the chromatin sample retains a small but significant anisotropy at the end of the bleach $\left(r_{\mathrm{b}}(0)=0.05\right)$; this anisotropy then decays with a time constant of about 100 microseconds.

If the $45 \mathrm{~kb}$ fibers were to behave like rigid rods with a mass per unit length of $170 \mathrm{kDa} / \mathrm{nm}$ and a diameter of $30 \mathrm{~nm}$ (S.P. Williams \& J.P. Langmore, unpublished results) their long axis and end-over-end rotational diffusion coefficients would be $4000 \mathrm{~s}^{-1}$ and $140 \mathrm{~s}^{-1}$, respectively. Thus, the 100 milrusecond decay time measured here is of the same order of magnitude as the long axis relaxation time of the digested fibers. The magnitude of $r_{b}(0)$ will primarily reflect internal motion of chromatin, rather than global reorientation. Internal motions are not expected to be significantly affected by interactions between chromatin molecules at concentrations as low as $150 \mu \mathrm{g} / \mathrm{ml}$. Therefore, because samples containing $30 \mathrm{~nm}$ digested chromatin fibers retain more anisotropy at the end of the bleach than do solutions containing naked DNA, it is probable that compaction of DNA into the $30 \mathrm{~nm}$ fiber acts to suppress internal motions which would normally be manifest in naked DNA.

\section{(f) Comparison of dynamics of quiescent and active nucle $i$}

We have studied the dynamies of both sea urchin and mudpuppy chromatin hecause several lines of evidence suggest that chromatin in sea urchin is less condensed than in mudpuppy. For example, one can visually detect this difference under the optical microscope. Nuclease digestion experiments show that sea urchin embryo chromatin is much more susceptible to enzymatic attack by micrococcal nuclease than mudpuppy erythrocyte chromatin (Jan Fronk, unpublished results). Of course, embryonic sea urchin nuclei are transcriptionally active while mudpuppy nuclei are inactive.

Here we have also demonstrated that sea urchin chromatin is more mobile than mudpuppy chromatin at physiological salt concentrations. We find that the mudpuppy anisotropy is static and equal to 0.2 . In contrast, the initial value of the sea urchin anisotropy is $0 \cdot 15$ and the $r_{\mathrm{b}}(t)$ value decays slightly. These dynamic differences are certainly consistent with the underlying biological differences and may indeed be a direet consequence of the latter.

\section{(g) Comparison with dynamics in congested gel solutions}

Recently, pFRAP has also been used to study the reorientational motion of naked DNA molecules in agarose gels (Scalettar et $a l ., 1990$ ). The gel experiments indicate that under moderately congested conditions the rapidly relaxing reorientational motions of naked DNA molecules are largely unperturbed. Specifically, it is found that in gels that are $5 \%$ agarose by weight, the initial anisotropy is $\leq 0.03$, or about 0.2 that measured in the unaggregated nuclei solutions. Hence, gels do not damp out many of the smaller amplitude motions of naked DNA that lead to depolarization during the bleach pulse. The relative immobility of the chromatin samples studied here seems to be a consequence of both the inherent rigidity of the chromatin fibers (section (b), above) and the concentration of fibers within the nucleus (section (d), above). 


\section{Conclusions}

We have monitored chromatin reorientation in intact nuclei as a function of salt concentration and local chromatin concentration. It is found that nuclear chromatin becomes progressively more inflexible as it undergoes a salt-induced conformational transition from an extended beads-on-astring structure to a compact $30 \mathrm{~nm}$ fiber. The folding process proceeds gradually as the salt concentration is varied and does not appear to reach any well-defined sub-physiological endpoint. Local increases in chromatin concentration, such as those that are induced by the side-by-side packing (aggregation) of chromalin, were found markedly to inhibit chromatin reorientation. Hence, the data presented here show that cellular chromatin, which is believed to exist primarily in the aggregated state, is highly immobile. We suggest that the immobilization is due to suppression of internal motion by high local concentration. In the future we hope to use the pFRAP technique to monitor chromatin dynamics directly in living cells and, thereby, to study the effects that mitosis and the binding of enzymes or drugs have on chromatin dynamies.

We gratefully acknowledge helpful conversations with James Abney and Nathan Hunt. We also received valuable assistance with software and the experimental apparatus from Robert Fulbright, Edward Hellen and Marisela Velez. Debbie Rankert, Shawn Williams, Claudius Vincenz, Jan Fronk and Graeme Tank deserve special thanks for preparation of the nuclei and chromatin samples, as well as electron microscopy and DNA electrophoresis. This work was supported by NIH grants GM41911 (J.E.H. and M.P.K.), NS14565 (D.A.) and GM27937 (J.P.L.); by NSF grants DMB8805296 (D.A.) and DIR8706052 (J.P.L.); by the Office of Energy Research, Office of Health and Environmental Research of DOE under contract DE AC03-76SF00098 (J.E.H. and M.P.K.); and by an appointment (B.A.S.) to the Alexander Hollaender Distinguished Postdoctoral Fellowship Program supported by DOE and administered by Oak Ridge Associated Universities.

\section{References}

Ashikawa, I., Kinosita, K., Ikegami, A., Nishimura, Y., Tsuboi, M., Watanabe, K., Iso, K. \& Nakano, T. (1983). Biochemistry, 22, 6018-6026

Ashikawa, I., Furuno, T., Kinosita, K., Ikegami, A., Takahashi, H. \& Akutsu, H. (1984). J. Biol. Chem. 259, 8338-8344.

Ashikawa, I., Kinosita, K., Tkegami, A., Nishimura, Y.\& Tsuboi, M. (1985). Biochemistry, 24, 1291-1297.

Athey, B. D., Smith, M. F., Williams, S. P., Rankert, D. \& Langmore, J. P. (1990). J. Cell. Biol. In the press.

Bevington, P. R. (1969). Data Reduction and Error Analysis in the Physical Sciences, pp. 237-239, MoGraw-Hill Book Co., New York.

Broersma, S. (1960). .J. Chem. Phys. 32, 1626-1631.

Butler, P. J. G. (1983). CRC Crit. Rev. Biochem. 15, $57-91$

Butler, P. J. G. \& Thomas, J. O. (1980). J. Mol. Biol. 140, $505-529$.
Ding, D., Rill, R. \& Van Holde, K. E. (1972). Biopolymers, 11, 2109-2124.

Erard, M., Das, G. C., de Murcia, G., Mazen, A., Pouyet, J., Champagne, M. \& Duane, M. (1979). Nucl. Acids Res. 6, 3231-3252.

Felsenfeld, G. \& MoGhee, J. D. (1986). Cell, 44, 375-377.

Finch, J. T. \& Klug, A. (1976). Proc. Nat. Acad. Sci., U.S.A. 73, 1897-1901.

Frank-Kamenetskii, M. D. (1981). Comments Mol. Cell. Biophys. 1, 105-114

Fulton, A. B. (1982). Cell, 30, 345-347.

Genest, D., Sabeur, G., Wahl, P. \& Auchet, J.-C. (1981). Biophys. Chem. 13, 77-87.

Hagerman, P. J. (1988). Annu. Rev. Biophys. Biophys. Chem. 17, 265-286.

Hard, T., Nielson, P. E. \& Norden, B. (1988). Eur Biophys. J. 16, 231-241.

Hurley, I., Osei-Gyimah, P., Archer, S., Scholes, P. \& Lerman, L. S. (1982). Biochemistry, 21, 4999-5009.

Kellenberger, E. (1987). Trends Biochem. Sci. 12 105-107.

Klevan, L., Armitage, I. M. \& Crothers, D. M. (1979). Nucl. Acids Res. 6, 1607-1616.

Langmore, J. P. \& Paulson, J. R. (1983). J. Cell Biol. 96 , 1120-1131

Livolant, F. \& Maestre, M. F. (1988). Biochemistry, 27, 3056-3068.

MacGregor, H. C. (1980). Heredity, 44, 3-35.

Magde, D., Elson, E. L. \& Webb, W. W. (1974) Biopolymers, 13, 29-61.

Magde, D., Zapalla, M., Knox, W. H. \& Nordlund, T. M. (1983). J. Phys. Chem. 87, 3286-3288.

McGhee, J. D., Rau, D. C., Charney, E. \& Felsenfeld, G. (1980). Cell, 22, 87-96.

McGhee, J. D., Nickol, J. M., Felsenfeld, G. \& Rau, D. C. (1983). Cell, 33, 831-841.

Nelson, W. G., Pienta, K. J., Barrack, E. R. \& Coffey, D. S. (1986). Annu. Rev. Biophys. Biophys. Chem. 15, $457-475$.

Olins, D. E. \& Olins, A. L. (1972). J. Cell Biol. 53 $715-736$

Paoletti, J., Magee, B. B. \& Magee, P. T. (1977) Biochemistry, 16, 351-357.

Paulson, J. R. \& Laemmli, .J. K. (1977). Cell, 12, 817-828.

Rill, R. L. (1986). Proc. Nat. Acad. Sci., U.S.A. 83, $342-346$.

Rill, R. L., Hilliard, P. R., Jr \& Lery, G. C. (1983). J. Biol. Chem. 258, 250-256.

Scalettar, B. A., Selvin, P. R., Axelrod, D., Hearst, J. E \& Klein, M. P. (1988). Biophys. J. 53, 215-226.

Scalettar, B. A., Hearst, J. E. \& Klein, M. P. (1989) Macromolecules, 22, 4550-4559

Scalettar, B. A., Selvin, P. R., Axelrod, D., Klein, M. P. \& Hearst, J. E. (1990). Biochemistry, 29, 4790-4798.

Schmitz, K. S. \& Schurr, J. M. (1973). Biopolymers, 12, $1543-1564$.

Schurr, R. L. \& Schurr, J. M. (1985). Biopolymers, 24 1931-1940.

Sen, D. \& Crothers, D. . M. (1986). Biochemistry, 25 , $1495-1503$.

Shibata, J. H., Fujimoto, B. S. \& Schurr, J. M. (1985). Biopolymers, 24, 1909-1930.

Shindo, H., McGhee, J. D. \& Cohen, J. S. (1980). Biopolymers, 19, 523-537.

Smith, M. F., Athey, B. D., Williams, S. P. \& Langmore, J. P. (1990). J. Cell. Biol. 110, 245-254.

Sorlie, S. S. \& Pecora, R. (1988). Macromolecules, 21, $1437-1449$. 
Suau, P., Bradbury, E. M. \& Baldwin, J. P. (1979). Eur. J. Biochem. 97, 593-602.

Thoma, F., Koller, Th. \& Klug, A. (1979). J. Cell. Biol. 83, 403-427.

Van Holde, K. E. (1989). Chromatin, Springer-Verlag, New York.

Velez, M. \& Axelrod, D. (1988). Biophys. J. 53, 575-591.

Wang, J., Hogan, M. \& Austin, R. H. (1982). Proc. Nat. Acad. Sci., U.S.A. 79, 5896-5900.
Williams, S. P., Athey, B. D., Mugiia, L. J., Schappe, R. S., Gough, A. H. \& Langmore, J. P. (1986). Biophys. J. 49, 233-248.

Widom, J. (1986). J. Mol. Biol. 190, 411-424.

Widom, J. (1989). Annu. Rev. Biophys. Biophys. Chem. 18, 365-395.

Workman, J. L. \& Langmore, J. P. (1985). Biochemisiry, 24, 4731-4738.

Edited by A. Klug 